\title{
Dynamics of clinical and neurological changes in patients with acute ischemic stroke in an open clinical study
}

\author{
Kamilla B. Srailova $^{1} * \mathbb{D}$, Bekmurat N. Raimkulov ${ }^{1}$, Erkyn S. Nurguzhaev ${ }^{1}$, Bakhtiyor G. Gafurov ${ }^{2}$, Gulsym B. Taukebayeva $^{3}$ \\ Received: 12 Dec 2020 \\ Published: 16 Sep 2021
}

\begin{abstract}
Background: Studies of treatment methods for patients with acute ischaemic stroke should include aetiological causes, concomitant pathology, and localisation of the lesion, and the extent of the lesion in the brain. The purpose of the study was to determine changes in clinical and neurological parameters in patients with ischaemic stroke in the acute period.

Methods: This is an open clinical study for which 240 patients were selected with an acute condition after an ischaemic stroke. All patients were divided into 4 groups (depending on the treatment). Clinical neurological examination and testing was performed upon admission to the hospital and upon discharge- after treatment. Electroencephalographic biofeedback (EEG-BFB) therapy was performed using a EEG-BSE device (Bio-Link). Data was processed according to the statistical method of experimental data assessment.

Results: To study the effectiveness of treatment upon acute ischaemic stroke, a comprehensive treatment system was developed, involving acupuncture, Qigong breathing exercises, and electroencephalographic biological feedback (EEG-BIOFEEDBACK), based mainly on the mechanisms of action. The study investigated the features of the acupuncture treatment in patients with ischaemic stroke during recovery. The authors noted the degree of effectiveness of EEG-BFB therapy, Qigong therapy, acupuncture, and standard treatment. Studies revealed that the development of ischaemic stroke begins gradually and at an early age.

Conclusion: It was concluded that the most effective method for treating the clinical and neurological manifestations of acute ischaemic stroke is EEG-BFB therapy, followed by acupuncture, Qigong therapy, and standard treatment.
\end{abstract}

Keywords: Treatment, Rehabilitation Programme, Subarachnoid Haemorrhages, Illness, Neurorehabilitation

Conflicts of Interest: None declared

Funding: None

*This work has been published under CC BY-NC-SA 1.0 license.

Copyright $₫$ Iran University of Medical Sciences

Cite this article as: Srailova KB, Raimkulov BN, Nurguzhaev ES, Gafurov BG, Taukebayeva GB. Dynamics of cllinical and neurological changes in patients with acute ischemic stroke in an open clinical study. Med J Islam Repub Iran. 2021 (16 Sep);35:119. https://doi.org/10.47176/mjiri.35.119

\section{Introduction}

Strokes are one of the global issues of modern medicine. They are observed in people in adulthood and old age more frequently, although there is a tendency to rejuvenation. On average, the frequency of stroke occurrence, according to many authors in different parts of the world, ranges from 1 to 4 and 1 to 5 cases per 1,000 population per year. In some $r$ egions, these figures can be higher and reach about 500,000 strokes per year (1-3). Among

Corresponding author: Kamilla B. Srailova, kamsrailova6068@nuos.pro

1. Department of Nervous Diseases with a Course of Neurosurgery, Asfendiyarov Kazakh National Medical University, Almaty, Republic of Kazakhstan

2. Department of Neurology, Tashkent Institute for Advanced Training of Doctors, Tashkent, Republic of Uzbekistan

3. Department of Clinical Pharmacology, Asfendiyarov Kazakh National Medical University, Almaty, Republic of Kazakhstan strokes, $70 \%$ to $80 \%$ are ischaemic and $20 \%$ to $25 \%$ are haemorrhagic, of which $5 \%$ are subarachnoid haemorrhages. Cerebral strokes are the most common brain diseases in adulthood and old age. The frequency of strokes varies in different regions of the world from 1 to 4 cases per 1,000 population per year, significantly increasing with aging. Consequences of strokes are treated in specialised departments of multidisciplinary clinics with the

$\uparrow$ What is "already known" in this topic:

Early rehabilitation reduces the development of complications in the acute period of stroke, such as hypokinesia and lack of exercise, complications of secondary pathological conditions, and impaired social and psychological depressive states.

$\rightarrow$ What this article adds:

It was found that acupuncture, Qigong therapy, and EEGBIOFEEDBACK therapy can be effectively used in patients with ischaemic stroke in the acute period with the correct selection of indications and treatment regimens 
presence of neurosurgical departments (4-7). The effectiveness of stroke outcomes depends on the timing of treatment initiation. In the first hours from the onset of the disease, the presence of a "therapeutic window" in the acute period and treatment refers to early rehabilitation. In perspective, early rehabilitation proves its effectiveness by the fact that only about $5 \%$ of patients become disabled and require out-patient care, and about $40 \%$ of patients return to their previous activities.

The rehabilitation \for the restoration of domestic, social skills, and restoration of lost motor and other functions can last for 2 years or more (8). In 2017, new global and European documents were developed, which established the basic elements of rehabilitation. The World Health Organization (WHO) document "Rehabilitation 2030" and "Report on Stroke in Europe" of the European Stroke Alliance (SAFE), as well as the ESO SAFE, Europe's Stroke Action Programme 2018-2030 highlight the need for and importance of stroke rehabilitation. The WHO "Global Stroke Bill of Rights" document also discusses assessment, rehabilitation, and social reintegration as a basic opportunity for a patient after a stroke (9).

Hypercapnic-hypoxic breathing exercises reduce neurological disorders (18 times on the Menves scale); namely, dysmotility, coordination dysfunction after ischaemic stroke in experiments on rats (10-12). The joint effect of hypoxia and hypercapnia (hypercapnic hypoxia) ultimately increases the body's resistance to acute hypoxia and effectively reduces neurological disorders in subtotal cerebral ischemia comparable with their isolated effect in animals $(13,14)$. It was established that the mechanisms of the neuroprotective effect of the combined effects of hypoxia and hypercapnia are performed at all levels: molecular, cellular, tissue, and body (15). Vestibular rehabilitation is important in the treatment of dizziness in patients with ischaemic stroke (16). The vestibular rehabilitation reduces the risk of falls and improves the patients' quality of life (17). Biofeedback (BFB) is one of the popular technologies in neurorehabilitation (18). With BFB (EEG), the functional state of the brain networks can be improved. The EEG is chosen due to the increased probability of effective treatment (19).

According to many studies, virtual reality technology demonstrates high efficiency in restoring the movement function and the manipulative function of the hand (20, 21). The bilateral use of innovative technologies with the BFB system of the Anika glove in patients after ischaemic stroke contributes to the effective recovery and improves the quality of life of these patients, as it improves fine motor skills of the hands (22). One of the methods of neurorehabilitation is electromyostimulation. Electrical stimulation creates an intense afferentation flow with deep paresis and plegium. Due to the activation of the primary sensory and motor zones, the mechanisms of neuroplasticity increase. Functional electrical stimulation is the most recommended treatment (23). Transcranial magnetic stimulation (TMS) and transcranial electrical stimulation (TES) are used in neurorehabilitation of central nervous system. These methods directly affect the areas of the brain that are responsible for lost functions, activating, or inhibiting them. TMS operates with an alternating magnetic field, and TES with a constant or alternating electric field (24-26). Achievements in modern medicine are reparative drugs. These include stem cells, gene therapy, monoclonal antibodies, extracts from biological tissues of animals, and other recovery accelerators (27).

The purpose of the study was to identify changes in clinical and neurological parameters in patients with acute ischaemic stroke.

\section{Methods}

To conduct an open clinical study, 240 patients (120 men and 120 women) who had an acute ischaemic stroke were involved. The mean age of patients was 40 to 70 years. All patients were divided into 4 groups, and received the following in combination with standard therapy: Group 1 ( $\mathrm{n}=48$ patients) received electroencephalographic biofeedback (EEG-BFB) therapy, Group 2 (56 patients) underwent additional acupuncture sessions, Group 3 (64 patients) received Qigong therapy, Group 4 (72 patients) made up the control group who received the standard therapy only.

Clinical neurological examination and testing was performed upon admission to the hospital and upon discharge - after treatment. The following criteria were used for the study: clarity of consciousness (15 points on the Glasgow coma scale), absence of gross cognitive impairment $(>20$ points on the short mental status assessment scale) and absence of severe speech disorders that prevent communication with the patient. The Spielberger-Hanin scale of situational (reactive) and personal anxiety was used to assess patients' mental health. Depending on the severity of the clinical effect, by the end of treatment, patients were divided into the following groups: (1) complete regression of neurological symptoms; (2) a significant improvement manifested by the disappearance of the overwhelming (more than $70 \%$ ) subjective and objective neurological manifestations at the end of treatment; (3) improvement and partial regression $(50 \%-70 \%)$ of neurological symptoms.

Acupuncture exposure was performed according to the second version of the inhibitory method. At the same time, at the beginning of the procedure, on the first session, 5 points were needled: Bai-hui (Vg20), Zu-san-li (E36), and He-gu (Gi4). These points were needled on the first, third, fifth, seventh, ninth, and eleventh days of treatment. Starting from the second procedure, the points of "wonderful meridians" were used: Zu-san-li (E36), He-gu (Gi4), and the next day, the points of the "wonderful meridians" Hou-si (3VI)-Shen-may (62VII); Zu-san-li (41XI)-Waiguan (5XI); Zhao-hai (6VIII)-Le-quue (7I); Gun-sun (4IV)-Ney-guan (6IX).

Acupuncture was used for additional treatment. The patients were previously examined. The pathogenesis of the disease, the lesion, and the leading syndrome were found, and topical diagnosis was performed. Based on this inspection, the skin projections of biologically active points were verified by proportional measurement using the standard method based on the atlases of classical manuals. In turn, this allowed mitigating the additional impact of 
biologically active points (BAP) on the body.

The EEG-BFB therapy was performed using an EEGBSE device (Bio-Link). The EEG-BFB device (Bio-Link) has an EEG helmet, which was worn on the head, then the primary registration of EEG waves with open and closed eyes was done and the matrix of transition states of EEG waves was determined. $\alpha, \beta, \tau$, and $\delta$ rhythms were automatically calculated. Then, one of the programmes was selected for moving the ball by force from the edge to the middle of the screen. The collected data were processed according to statistical method of experimental data assessment using Excel (Microsoft).

All procedures involving human participants were performed in accordance with the ethical standards of the institutional and national research committee and with the 1964 Helsinki declaration and its later amendments or comparable ethical standards. The Ministry of Health and Social Development of the Republic of Kazakhstan approved this study under the Protocol No. 18 dated December 27, 2016, and this study has been described in clinical protocols (28).

\section{Results}

Acupuncture Treatment in Patients With Ischemic Stroke in the Recovery Period

Qigong is a wellness system that aims to prevent and treat diseases, slow aging, and prolong life span. Selfcontrolled Qigong therapy is a method of controlling the body with special exercises that stabilize the condition most favourable for overcoming diseases. Qigong is a special type of auto-training in the treatment and prevention of various diseases. It comprises a system of exercises, during which harmony of the heart (xin), consciousness (izhi), and breathing is achieved, jing, qi, and shen are strengthened, true qi (zhenqi) is strengthened, the internal functions of the body are ordered, thereby improving immunity. Breathing exercises include two interconnected components: physical and respiratory exercises, and psychological and meditative exercises. Meditation consists of focusing the attention of patients on a given visual image or a specific area of the Dan-Tian body. Respiratory systems are divided into various systems: "Neyan-gun"- exercises for prevention from the inside; the Jiang-chuang-gong system- exercises for general strengthening; Baojiang-gong system- exercises for supporting health.

Patients with severe disease should perform exercises while lying or sitting; in case of mild illnesses - in a standing position, moving in steps as well as by the method of tai chi (qigong of the Great Reach). Tai chi gymnastics in the form of dosed physical exercises are a factor that restores the autonomic functions and activity of the cardiovascular system. Tai chi gymnastics has a stimulating effect on the metabolism through the nervous system due to the humoral regulation of tissue metabolism. This leads to a decrease in the energy expenditure of substances in the body during muscle activity. There is also a stimulating effect on the endocrine, reticuloendothelial, immune systems, and enzymatic activity.
Qigong therapy: breathing (static) exercises are performed in the following position: lying down; sitting; standing. The posture is selected individually depending on the patient's physical condition and comfort. Technique: (1) lying position: the patient lies on a flat surface on their back, legs are straightened, hands are placed along the hips; (2) sitting position: the patient sits on a chair, the head is straight, the spine is straightened, the legs are bent at the knees, the palms on the hips; (3) standing position: legs shoulder-width apart, slightly bent at the knees, arms bent in front of the chest, torso relaxed, eyes closed. Exercises are performed in one of the selected positions. The patient inhales and exhales freely, calmly, easily, and slowly. The tongue is pressed to the palate. While breathing, the patient mentally focuses on the YinTang point (located between the eyebrows). The duration of the exercise is 3 to 5 minutes. At the end, the patient takes a deep breath, then exhales and opens their eyes. After the static exercise, the patient proceeds to the second part of Qigong therapy - self-massage.

Self-massage technique: the patient is recommended to focus on their actions, then take a comfortable position. The palms of the hands rub the entire body, starting from the face, then going to the scalp, neck, upper limbs, trunk, and ending with the level of the inguinal fold. After this, rubbing of the tips of the toes, feet, calves, thighs to the level of the inguinal fold. Then, the patient switches to joint gymnastics, which is a part of self-massage. Circular rotation is performed in all groups of joints of the upper and lower extremities, cervicial, thoracic, lumbar spine. Circular rotation is performed up to 6 to 12 times clockwise and counterclockwise. The next stage of selfmassage is light stroking of the liver, abdomen, lumbar region. Technique: the palm of the right hand is fixed on the area where the patient is going to perform selfmassage, the palm of the left hand is set at the same level, only at the back. Slow stroking is performed 24 times clockwise and 18 times counterclockwise. It is this amount of stroking that corresponds to the biological cycle of the flow of energy in the body. The treatment course using Qigong therapy is 10 to 12 sessions. Additionally, elements of tai chi gymnastics were used in patients with astheno-neurotic syndrome as follows:

- Exercise 1. Initial position: legs shoulder-width apart, torso straightened, arms at side, eyes at primary position. The patient slowly raises hands to the shoulder level with palms down, then slightly squatting and bending at the knee and elbow joints, the hands drop down. Then, the hands are raised up to the sides with a return to their original position. This exercise is performed 6 to 12 times.

- Exercise 2 is called "hands like a cloud" (yun-shou). Initial position: feet shoulder-width apart, the left hand is at eye level with the palm inward, the right hand is palm down parallel to the floor. A turn in a semicircle to the left begins. At the same time, the patient slowly and smoothly puts the left foot on the heel. The eyes are fixed on the left palm, the patient takes a step, putting the right foot to the left. The eyes are fixed on the right hand, which is set as the left, with the palm facing the patient. The left hand is in the position that the right hand previously held, palm 
down parallel to the floor. In this position, a semicircle is made. Then the exercise is repeated in the other direction. This is done 6-12 times.

- Exercise 3. Starting position: feet shoulder-width apart. The palms of both hands are put on the stomach, the body is straightened, eyes at primary position. The patient turns their head to the right while the hands slide up and down along the waist. The right hand is wound behind the back, gliding along the lower back with the dorsum while the left palm glides along the stomach. The patient looks at the left heel (on inhale) for 5 seconds. Slowly, on inhale, the patient returns to the initial position. The same is performed in the opposite direction. The exercise is repeated 6-12 times.

- Exercise 4. Initial position: legs spread wide, slightly crouching, eyes at primary position, body straightened. Hands go slowly to the right side (inhale); the index finger is at the level of the eyebrows, the left hand stops opposite the waist in front, with fingers up, palms forward. Then, slowly, on exhale, the patient returns to the initial position. The exercise is performed 6-12 times in each direction. At the end of the exercises, the patient puts the right foot to the left so that both legs are shoulder-width apart.

- Exercise 5. Initial position: the left leg is put forward, in line with the right leg, the body is straightened, eyes at primary position. Hands are put down, slightly bent, palms facing forward. With a slight sliding movement, the patient pulls the right foot forward towards the left foot, with a sliding movement. At the same time, the patient raises their hands up on inhale. Feet together, hands lower down on exhale. Next, the patient repeats the exercise in the mirrored position. The exercise is performed 6-12 times.

- Exercise 6. Initial position: legs shoulder-width apart, left hand clenched into a fist, put on waist, right hand is lowered down. The right hand rises on inhale, then goes behind the back. Mentally, a circle is drawn with a hand.
This exercise is performed slowly, calmly, 6-8 times with each hand.

- Exercise 7. Initial position: legs shoulder-width apart, body straightened, arms at side. On inhale, both hands slowly rising up to shoulder level, palms down, then hands gradually lower to their initial position. The exercise is performed 6 to 8 times.

At the end of the exercises, a self-massage of acupuncture points $\mathrm{He}-\mathrm{Gu}, \mathrm{Zu}-\mathrm{San}-\mathrm{Li}$, and San-Yin-Jiao is performed. Technique: the patient is in a comfortable position, with the thumb or forefinger kneading on acupuncture points. The movements are helical, with a displacement of the skin, clockwise, then counter-clockwise - 6-8 times each. The clinical effect was evaluated based on the analysis of subjective and objective symptoms described in chapter II, and the results of electrophysiological research methods (REG, EEG) performed during treatment using the above complex.

\section{Comparative Characteristics of the Effectiveness of} EEG-BFB Therapy and Qigong Therapy

Characterisation of a computerised EEG-BFB complex was used with EEG-BFB therapy as additional treatment in the Group 1, during morning hours, for 10-20 minutes. Persons with decompensated diseases, epilepsy, Parkinson's syndrome were excluded. The course of treatment included 10-12 procedures performed as a single procedure daily (Tables 1-2).

General weakness was inherent in all groups of patients. The Barre test was positive upon admission and discharge in $2.1 \%$ of patients in group 1; group 2 in $14.3 \%$ before and $3.6 \%$ after treatment; group 3 in $3.2 \%$ with complete regression; and in group 4 in $5.6 \%$ with an increase to $9.7 \%$. Before treatment, anisoreflexia was observed in $35.5 \%$ of patients in group 1; in group 2 in $30.3 \%$; in group 3 in $21.8 \%$; and in group 4 in $58.2 \%$. After treat-

Table 1. The Matrix of Transition Probabilities of EEG Waves in the Main Group of Patients

\begin{tabular}{|c|c|c|c|c|}
\hline \multirow[t]{2}{*}{ Open Eyes } & \multicolumn{2}{|c|}{ Before Treatment } & \multicolumn{2}{|c|}{ After Treatment } \\
\hline & Right & Left & Right & Left \\
\hline$\alpha$ & 0.801 & 1.614 & 0.701 & 1.930 \\
\hline$\beta$ & 1.228 & 1.390 & 1.403 & 1.653 \\
\hline$\tau$ & 0.785 & 1.002 & 0.838 & 0.762 \\
\hline$\delta$ & 0.668 & 0.352 & 0.428 & 0.168 \\
\hline \multirow[t]{2}{*}{ Closed eyes } & \multicolumn{2}{|c|}{ Before treatment } & \multicolumn{2}{|c|}{ After treatment } \\
\hline & Right & Left & Right & Left \\
\hline $\bar{a}$ & 1.480 & 1.398 & 1.390 & 1.613 \\
\hline $\mathrm{b}$ & 1.664 & 1.658 & 1.778 & 0.865 \\
\hline $\mathrm{t}$ & 0.740 & 0.767 & 0.733 & 0.615 \\
\hline d & 0.196 & 0.166 & 0.432 & 0.354 \\
\hline
\end{tabular}

Table 2. The Matrix of Transition Probabilities of EEG Waves in the Control Group

\begin{tabular}{|c|c|c|c|c|}
\hline \multirow[t]{2}{*}{ Open Eyes } & \multicolumn{2}{|c|}{ Before Treatment } & \multicolumn{2}{|c|}{ After Treatment } \\
\hline & Right & Left & Right & Left \\
\hline $\mathrm{a}$ & 0.701 & 1.245 & 0.246 & 1.614 \\
\hline $\mathrm{b}$ & 1.778 & 1.650 & 1.230 & 1.651 \\
\hline $\mathrm{t}$ & 0.826 & 0.766 & 0.786 & 0.762 \\
\hline d & 0.486 & 0.768 & 1.688 & 0.864 \\
\hline \multirow[t]{2}{*}{ Closed eyes } & \multicolumn{2}{|c|}{ Before treatment } & \multicolumn{2}{|c|}{ After treatment } \\
\hline & Right & Left & Right & Left \\
\hline $\bar{a}$ & 1.498 & 1.392 & 1.490 & 1.248 \\
\hline $\mathrm{b}$ & 1.230 & 1.394 & 0.448 & 0.604 \\
\hline $\mathrm{T}$ & 0.768 & 0.836 & 1.120 & 1.115 \\
\hline $\mathrm{D}$ & 0.432 & 0.354 & 0.870 & 0.486 \\
\hline
\end{tabular}


ment, these numbers in all 4 groups of patients were $18.8 \% ; 14.3 \% ; 9.8 \%$; and $40.3 \%$, respectively. In group 1 , hemi-hypoesthesia developed in $58.3 \%$ of patients and hemiparesis in $20.8 \%$ of patients; in group 2 in $42.9 \%$ and $21.4 \%$ of patients, respectively; in group 3 in $31.3 \%$ and $29.7 \%$ of patients, respectively; and in group 4 in $47.2 \%$ and $67.7 \%$, respectively. After treatment, hemihypoesthesia and hemiparesis persisted in $27.1 \%$ and $50.0 \%$ of patients in group 1; in group 2 in $17.9 \%$ and $32.1 \%$, respectively; in group 3 in $31.3 \%$ and $12.59 \%$, respectively; and in group 4 in $18.1 \%$ and $41.7 \%$, respectively. Numbness of the extremities was observed in $56.3 \%$ of patients in group 1 before treatment and in $12.5 \%$ after treatment; in group 2 in $42.9 \%$ before and $14.3 \%$ after treatment; in group 3 in $21.9 \%$ before and $9.2 \%$ after treatment; and in group 4 in $43.1 \%$ and $13.9 \%$, respectively.

Instability in the Romberg position with impaired finger test was found in patients of group 1 in $29.2 \%$ and $39.6 \%$ before and $20.8 \%$ and $20.8 \%$ after treatment; in group 2 , in $46.4 \%$ and $51.8 \%$ before and $16.1 \%$ and $14.3 \%$ after treatment; in group 3, in $53.1 \%$ and $45.3 \%$ before and in $23.4 \%$ and $21.9 \%$ after treatment; and in group 4 in $29.2 \%$ and $31.9 \%$ before and in $25 \%$ and $13.9 \%$ after treatment. Unsteadiness when walking was detected upon admission in $31.3 \%$ of patients in group 1 and in $4.2 \%$ of patients upon discharge; in group 2 in $25 \%$ upon admission and in $25 \%$ upon discharge; in group 3 in $42.2 \%$ upon admission and in $9.2 \%$ upon discharge; in group 4 in $23.6 \%$ upon admission and in $12.5 \%$ upon discharge. Pathological symptoms of Babinsky and oral automatism were noted before treatment in $50 \%$ of patients in group 1 ; in $4.8 .2 \%$ of patients in group 2 ; in $42 \%$ of patients in group 3; and in $63.9 \%$ of patients in group 4 . After treatment, it was noted in $27.1 \%$ of patients in group 1; in $35.7 \%$ of patients in group 2 ; in $20.4 \%$ of patients in group 3 , and in $54.1 \%$ of patients in group 4.

When considering the effect of various treatment complexes in patients with acute ischaemic stroke in terms of clinical manifestations, a pronounced regression of headaches was noted in groups 1 to 3 who received EEG-BFB therapy, acupuncture, and Qigong therapy sessions with a decrease from $45.8 \%, 62.5 \%, 65.6 \%$, respectively, to $4.2 \%, 7.1 \%$, and $0 \%$, respectively. In group 4 who received standard therapy, the regression went from $59.7 \%$ before treatment to $40.3 \%$ after treatment. The headache regression efficiency was $43.6 \%$ in group $1,55.4 \%$ in group $2,100 \%$ in group 3 , and $19.4 \%$ in group 4 . The standard treatment, despite the ongoing antihypertensive therapy, is ineffective against headache. A notable improvement is observed in the group of patients who received Qigong, acupuncture, and EEG-BFB therapy. In addition, such a sign as nausea, was observed in $41.7 \%$, $48.2 \%, 31.3 \%$, and $23.6 \%$ of patients in groups 1 to 4 , respectively, upon admission. During the treatment, nausea persisted in $1.8 \%$ of patients in group $2 ; 4.2 \%$ in group 4; however, no nausea was observed in groups 1 and 3. The effectiveness of the regression of nausea in groups 1 and 3 was $100 \%$, meaning that it was absent upon discharge. In groups 2 and 4 , nausea decreased to
$46.2 \%$ and $19.3 \%$, respectively. Comparative characteristics of clinical manifestations patients are presented in Table 3.

The effectiveness of EEG-BFB therapy and Qigong therapy for regression of nausea and, to a lesser extent, the use of acupuncture and standiard treatment are noted. If nausea at admission was present in $22.9 \%, 33.9 \%, 32.8 \%$, and $15.3 \%$ of patients of the 4 groups, then after treatment, then nausea persisted in $3.6 \%$ of patients in the 2 group and $6.9 \%$ of the controls. The effectiveness of the regression of pathological symptoms in the form of nausea was $100 \%$ in groups 1 and 3 . In groups 2 and 4, it was $30.2 \%$ and $8.4 \%$, respectively. From which it follows that an effective effect on nausea is noted when applying treatment methods in the form of EEG-BFB therapy and Qigong gymnastics. The use of acupuncture and standard treatment is less effective. It should also be noted that high incidence of dizziness upon admission was observed in $68.8 \%, 67.9 \%, 67.2 \%$, and $54.2 \%$ of patients in all 4 groups. After treatment, dizziness persisted in patients in all 4 groups in $4.2 \%, 8.9 \%, 1.6 \%$, and $19.4 \%$ of cases. The effectiveness of the regression of pathological dizziness was as follows in different groups: group 1: 64.6\%; group 2: $59 \%$, group 3: $65.6 \%$, and group 4: $34.8 \%$. In this case, there was a low efficiency of stopping dizziness by standard treatment methods in group 4.

At the initial examination of patients, it was noted that they were inhibited in $58.3 \%, 73.2 \%, 45.3 \%$, and $54.2 \%$ of cases in all 4 groups, respectively. However, after the treatment, these figures dropped to $6.3 \%$ in group $1,1.8 \%$ in group $2,37.5 \%$ in group 4 , and these manifestations were absent in group 3 . The efficiency of regression of the inhibition state was $52 \%$ in group $1,71.8 \%$ in group 2, $100 \%$ in group 3 , and $7.8 \%$ in group 4 . The condition in the form of general weakness at admission was observed in all 4 groups in $3 \%, 28.6 \%, 23.4 \%$, and $11.1 \%$ of patients, respectively. At discharge, these manifestations were absent in all 4 groups. The effectiveness of the regression of general weakness was $100 \%$ in all 4 groups. The use of complex methods and a standard method of treatment contributes to a complete regression of general weakness.

Decreased memory for current events is often the cause of cognitive impairment and their manifestations were noted in $12.5 \%, 16.1 \%, 25.0 \%$, and $13.9 \%$ in all 4 groups of patients upon admission, respectively. Despite the provisional treatment at discharge, memory did not recover in $2.1 \%, 3.6 \%$, and $12.5 \%$ of cases in groups 1,2 , and 4 . The efficiency of memory recovery in group 1 was $10.4 \%$, in group 2 was $12.7 \%$, in group 3 was $100 \%$; and in group 4 was $1.4 \%$. In this study, Qigong therapy contributes to the complete restoration of memory for patients, partially restored memory with standard treatment, and to a lesser extent in patients who received EEG-BFB therapy or acupuncture. Manifestations in the form of weakness in 1 or 2 limbs were observed in $77.1 \%$ of patients in group 1 ; in $50 \%$ in group 3 , and in $45.9 \%$ in group 4 . After treatment, weakness remained in $1.6 \%$ of patients in group 3 and in $22.4 \%$ of patients in group 4 . The effectiveness of regression of weakness in the limbs was $100 \%$ in group 1 , 
Dynamics of clinical and neurological changes in patients with acute ischaemic stroke

Table 3. Clinical and Neurological Indicators in a Group of Patients Treated With EEG-BFB, Acupuncture, Qigong Therapy, and in the Control Group

\begin{tabular}{|c|c|c|c|c|c|c|c|c|c|c|c|c|c|c|c|c|}
\hline \multirow{4}{*}{$\begin{array}{l}\text { Symptom } \\
\text { Names }\end{array}$} & \multicolumn{16}{|c|}{ Group of Patients } \\
\hline & \multicolumn{4}{|c|}{ First group EEG-BFB - 48} & \multicolumn{4}{|c|}{ Second group acupuncture -56} & \multicolumn{4}{|c|}{ Third group Qigong - 64} & \multicolumn{4}{|c|}{ Total - $72(100 \%)$} \\
\hline & \multicolumn{2}{|c|}{$\begin{array}{l}\text { Before } \\
\text { treatment }\end{array}$} & \multicolumn{2}{|c|}{$\begin{array}{c}\text { After } \\
\text { treatment }\end{array}$} & \multicolumn{2}{|c|}{$\begin{array}{l}\text { Before } \\
\text { treatment }\end{array}$} & \multicolumn{2}{|c|}{$\begin{array}{c}\text { Before } \\
\text { treatment }\end{array}$} & \multicolumn{2}{|c|}{$\begin{array}{c}\text { Before } \\
\text { treatment } \\
\end{array}$} & \multicolumn{2}{|c|}{$\begin{array}{c}\text { Before } \\
\text { treatment }\end{array}$} & \multicolumn{2}{|c|}{$\begin{array}{l}\text { Before } \\
\text { treatment }\end{array}$} & \multicolumn{2}{|c|}{$\begin{array}{c}\text { Before } \\
\text { treatment }\end{array}$} \\
\hline & Quantity & $\%$ & Quantity. & $\%$ & Quantity & $\%$ & Quantity & $\%$ & Quantity & $\%$ & Quantity & $1 \%$ & Quantity & $\%$ & Quantity & $\%$ \\
\hline Headache & 22 & 45.8 & 2 & 4.2 & 35 & 62.5 & 4 & 7.1 & 42 & 65.6 & & & 43 & 59.7 & 29 & 40.3 \\
\hline Nausea & 20 & 41.7 & & & 27 & 48.2 & 1 & 1.8 & 20 & 31.3 & & & 17 & 23.6 & 3 & 4.2 \\
\hline Nausea & 11 & 22.9 & & & 19 & 33.9 & 2 & 3.6 & 21 & 32.8 & & & 11 & 15.3 & 5 & 6.9 \\
\hline Dizziness & 33 & 68.8 & 2 & 4.2 & 38 & 67.9 & 5 & 8.9 & 43 & 67.2 & 1 & 11.6 & 39 & 54.2 & 14 & 19.4 \\
\hline Retardation & 28 & 58.3 & 3 & 6.3 & 41 & 73.2 & 1 & 1.8 & 29 & 45.3 & & & 39 & 54.2 & 27 & 37.5 \\
\hline $\begin{array}{l}\text { Noise in the } \\
\text { head }\end{array}$ & & & & & & & 1 & 1.8 & & & & & & & & \\
\hline $\begin{array}{l}\text { General weak- } \\
\text { ness }\end{array}$ & 16 & 33.3 & & & 16 & 28.6 & & & 15 & 23.4 & & & 8 & 11.1 & & \\
\hline $\begin{array}{l}\text { Memory } \\
\text { impairment }\end{array}$ & 6 & 12.5 & 1 & 2.1 & 9 & 16.1 & 2 & 3.6 & 16 & 25.0 & & & 10 & 13.9 & 9 & 12.5 \\
\hline Irritability & & & & & 3 & 5.4 & & & 9 & 14.1 & & & 2 & 2.8 & 2 & 2.8 \\
\hline $\begin{array}{l}\text { One limb } \\
\text { weakness }\end{array}$ & 1 & 2.1 & & & 4 & 7.1 & & & 5 & 7.8 & 1 & 11.6 & 3 & 4.2 & 2 & 2.8 \\
\hline Limb weakness & 36 & 75.0 & & & & & & & 27 & 42.2 & 11 & 17.2 & 30 & 41.7 & 14 & 19.4 \\
\hline Dysarthria & 36 & 75.0 & 9 & 18.8 & 27 & 48.2 & 13 & 23.2 & 44 & 68.8 & 4 & 6.3 & 55 & 76.4 & 17 & 23.6 \\
\hline $\begin{array}{l}\text { Language } \\
\text { deviation }\end{array}$ & 10 & 20.8 & 4 & 8.3 & 12 & 21.4 & 3 & 5.4 & 9 & 12.5 & 2 & 3.2 & 14 & 19.4 & 14 & 19.4 \\
\hline $\begin{array}{l}\text { Weakness of } \\
\text { the act of con- } \\
\text { vergence }\end{array}$ & 6 & 12.5 & 3 & 6.3 & 12 & 21.4 & & & 17 & 26.6 & 2 & 3,2 & 15 & 20.8 & 1 & 1.4 \\
\hline Anisocoria & 1 & 2.1 & 2 & 4.2 & 2 & 3.6 & & & 3 & 4.9 & & & & & & \\
\hline Double vision & & & & & & & & & & & 10 & 15.6 & 4 & 5.6 & 1 & 1.4 \\
\hline Fine nystagmus & 12 & 25.0 & 9 & 18.8 & 17 & 30.1 & 7 & 12.5 & 17 & 26.6 & 11 & 17.2 & 16 & 22.2 & 9 & 12.5 \\
\hline $\begin{array}{l}\text { Coarse nystag- } \\
\text { mus }\end{array}$ & 2 & 4.2 & 1 & 2.1 & 8 & 14.3 & 1 & 1.8 & 5 & 7.8 & 2 & 3,2 & 2 & 2.8 & 2 & 2.8 \\
\hline $\begin{array}{l}\text { Hemianopsia } \\
\text { homonymous }\end{array}$ & 1 & 2.1 & 1 & 2.1 & 2 & 3.6 & 2 & 3.6 & 2 & 3,2 & 1 & 11.6 & 3 & 4.2 & 6 & 8.3 \\
\hline $\begin{array}{l}\text { Nasolabial fold } \\
\text { asymmetry }\end{array}$ & 24 & 50.0 & 15 & 31.3 & 22 & 39.3 & 17 & 30.1 & 24 & 37.5 & 19 & 29.7 & 31 & 43.1 & 27 & 37.5 \\
\hline $\begin{array}{l}\text { Paresis } 7 \text { and } 12 \\
\text { pairs }\end{array}$ & 6 & 12.5 & 2 & 4,2 & 11 & 19.6 & 6 & 10.7 & 8 & 12.5 & 4 & 6.3 & 17 & 23.6 & 26 & 36.1 \\
\hline $\begin{array}{l}\text { Bulbar Disor- } \\
\text { ders }\end{array}$ & 4 & 8.3 & & & 3 & 5.4 & 1 & 1.8 & 5 & 7.8 & 1 & 11.6 & 10 & 13.9 & 8 & 11.1 \\
\hline $\begin{array}{l}\text { The recovery of } \\
\text { tendon reflexes }\end{array}$ & 17 & 35.5 & 9 & 18.8 & 17 & 30.3 & 8 & 14.3 & 14 & 21.8 & 6 & 9.8 & 27 & 37.5 & 20 & 27.8 \\
\hline $\begin{array}{l}\text { Muscle tone } \\
\text { increased }\end{array}$ & 7 & 14.6 & 1 & 2.1 & 11 & 19.6 & 1 & 1.8 & 11 & 17.2 & 3 & 4.9 & 15 & 20.8 & 5 & 6.9 \\
\hline Anisoreflexia & 17 & 35.5 & 9 & 18.8 & 17 & 30.3 & 8 & 14.3 & 14 & 21.8 & 6 & 9.8 & 38 & 52.8 & 29 & 40.3 \\
\hline $\begin{array}{l}\text { Hemi- } \\
\text { hypoesthesia }\end{array}$ & 28 & 58.3 & 13 & 27.1 & 24 & 42.9 & 10 & 17.9 & 20 & 31.3 & 9 & 12.5 & 34 & 47.2 & 13 & 18.1 \\
\hline $\begin{array}{l}\text { Hemi- } \\
\text { hyperesthesia }\end{array}$ & & & & & & & 1 & 1.8 & & & 1 & 11.6 & 4 & 5.6 & 4 & 5.6 \\
\hline Hemiparesis & 10 & 20.8 & 24 & 50.0 & 12 & 21.4 & 18 & 32.1 & 19 & 29.7 & 14 & 21.9 & 4 & 5.6 & 4 & 5.6 \\
\hline Hemiplegia & & & & & & & 3 & 5.4 & 2 & 3.2 & & & 48 & 66.7 & 30 & 41.7 \\
\hline Finger tremor & & & 1 & 2.1 & & & & & & & & & & & & \\
\hline $\begin{array}{l}\text { Instability in } \\
\text { the Romberg } \\
\text { position }\end{array}$ & 14 & 29.2 & 10 & 20.8 & 26 & 46.4 & 9 & 16.1 & 34 & 53.1 & 15 & 23.4 & 21 & 29.2 & 18 & 25 \\
\hline $\begin{array}{l}\text { Finger nasal } \\
\text { test }\end{array}$ & 19 & 39.6 & 10 & 20.8 & 29 & 51.8 & 8 & 14.3 & 29 & 45.3 & 14 & 21.9 & 23 & 31.9 & 14 & 19.4 \\
\hline $\begin{array}{l}\text { Symptom of } \\
\text { Babinsky }\end{array}$ & 22 & 45.8 & 12 & 25.0 & 22 & 39.3 & 16 & 28.6 & 21 & 32.8 & 12 & 18.8 & 37 & 51.4 & 33 & 45.8 \\
\hline $\begin{array}{l}\text { Symptoms of } \\
\text { oral automatism }\end{array}$ & 2 & 4.2 & 1 & 2.1 & 5 & 8.9 & 4 & 7.1 & 6 & 9.2 & 1 & 11.6 & 9 & 12.5 & 6 & 8.3 \\
\hline Barre & 1 & 2.1 & 1 & 2.1 & 8 & 14.3 & 2 & 3.6 & 2 & 3.2 & & & 4 & 5.6 & 7 & 9.7 \\
\hline Precariousness & 15 & 31.3 & 2 & 4.2 & 14 & 25.0 & 1 & 1.8 & 27 & 42.2 & 5 & 7.8 & 17 & 23.6 & 9 & 12.5 \\
\hline $\begin{array}{l}\text { Numbness of } \\
\text { limbs }\end{array}$ & 27 & 56.3 & 6 & 12.5 & 24 & 42.9 & 8 & 14.3 & 14 & 21.9 & 6 & 9.2 & 31 & 43.1 & 10 & 13.9 \\
\hline
\end{tabular}

$44.3 \%$ in group 3 , and $23.4 \%$ in group 4 .

It is noted that the use of EEG-BFB training is most effective for weakness in the limbs, followed by Qigong therapy and standard treatment. An increase in tone in the limbs was noted before treatment in all 4 groups of patients and accounted for $14.6 \%, 19.6 \%, 17.2 \%$, and $20.8 \%$, respectively. After treatment, these data were $2.1 \%, 1.8 \%, 4.9 \%$, and $6.9 \%$, respectively. The effectiveness of regression of increased muscle tone in the limbs in group 1 was noted in $12.5 \%$ of patients, in group 2 in $17.8 \%$, in group 3 in $12.3 \%$, and in group 4 in $13.9 \%$. The study noted the effectiveness of treatment from a decrease in muscle tone caused by ischaemic stroke with the acupuncture method, the standard method of treatment, EEGBFB training, and Qigong therapy. The Barre test was positive both upon admission and discharge in $2.1 \%$ of patients in group 1 without regression; in group 2 in $14.3 \%$ before and $3.6 \%$ after treatment; in group 3 complete regression in 3.2\%; and in group 4 in $5.6 \%$ with an increase to $9.7 \%$. Also, $100 \%$ effectiveness of the treatment and regression of the Barre test was noted upon the use of Qigong therapy, 10.7\% acupuncture, no regression during EEG-BFB training, and an increase of $+4.1 \%$ in group 4 after treatment. 
Dysfunction of the IX and X pairs of cranial nerves led to dysarthria and speech impediment. Upon admission and after discharge, these manifestations were observed in patients of group 1 in $75 \%$ and $20.8 \%$ of cases; in group 2 in $48.2 \%$ and $21.4 \%$; in group 3 in $68.8 \%$ and $12.5 \%$; in group 4 in $76.4 \%$ and $19.4 \%$, respectively. After treatment, these data were $18.8 \%$ and $8.3 \%$ in group $1 ; 23.2 \%$ and $5.4 \%$ in group $2 ; 6.3 \%$ and $3.2 \%$ of cases in group 3 ; and in $19.4 \%$ of group 4 the speech impediment remained unchanged after treatment. The effectiveness of the regression of pathological symptoms in the form of dysarthria was $56.2 \%$ in group $1 ; 25 \%$ in group $2 ; 62.5 \%$ in group 3; and $100 \%$ in group 4 . Violations in the form of speech impediment in terms of regression efficiency amounted to $12.5 \%$ in group $1,16 \%$ in group 2 , and $9.3 \%$ in group 3 and remained unchanged in group 4 (19.4\%). When comparing the effectiveness of treatment, a complete regression of clinical manifestations in the form of dysarthria was observed in all 4 groups of patients, with the first place occupied by group 4 (standard treatment), then group 2 and 1 (Qigong therapy and EEG-BFB therapy, respectively). In the last place is group 3 (acupuncture).

Regression of the clinical sign in the form of language deviation is noted in the group of patients who received acupuncture, EEG-BFB, Qigong therapy, and the manifestations in the control group remained unchanged. In group 1 , the effect of treatment complexes on impairment of vision in the form of weakness upon convergence, nystagmus, hemianopsia, anisocoria, and double vision was observed in $45.9 \%$ of patients before and $33.5 \%$ after treatment; in group 2, these indicators were $73 \%$ before and $17.9 \%$ after treatment; in group 3 it was $69.9 \%$ before and $40.8 \%$ after treatment; and in group 4 it was $33.4 \%$ before and $26.4 \%$ after treatment. The regression efficiency of the complex of visual impairment was as follows in the 4 groups: in group 1:12.4\%; group 2, 63.1\%, group 3: $29.1 \%$; and group $4: 7 \%$. The regressive scale of the effectiveness of medical complexes is in the following order: group 2: acupuncture method; group 3: Qigong therapy; group 1: EEG-BFB therapy; and group 4: control with standard treatment. Notably, none of the treatment complexes contributed to the complete regression of these clinical manifestations.

Analysis of clinical manifestations in the form of asymmetry of the nasolabial folds with a skewed face, paresis of 7 and 12 pairs of craniocerebral, and bulbar disorders of the cranial nerves was observed in patients of group 1 in $70.8 \%$ and $35.5 \%$ after treatment; in group 2 in $64.3 \%$ before and $42.6 \%$ after treatment; in group 3 in $57.8 \%$ before and $37.6 \%$ after treatment; and in group 4 in $80.6 \%$ and $84.7 \%$ after treatment. Effective regression of clinical manifestations of ischaemic stroke was identified in $35.3 \%$ of patients in group $1 ; 21.7 \%$ in group $2 ; 20.02 \%$ in group 3; and it was increased by $4.1 \%$ in group 4 . When comparing the effectiveness of medical complexes on the regression of clinical manifestations, there was a higher efficiency of EEG-BFB treatment, acupuncture, and Qigong therapy in the groups 1 to 3, respectively. Therapy in group 4 was less effective. Before treatment, cortical disturbances in the form of anisoreflexia were observed in group 1 in $35.5 \%$, in group 2 in $30.3 \%$, in group 3 in $21.8 \%$, and in group 4 in $58.2 \%$. After treatment, these numbers were $18.8 \%, 14.3 \%, 9.8 \%$, and $40.3 \%$ in groups 1 to 4 , respectively. The anisoreflexia regression efficiency was $3 \%, 16 \%, 12.5 \%$, and $17.9 \%$, respectively.

In group 1, hemi-hypoesthesia was detected in $58.3 \%$ of patients, hemiparesis in $20.8 \%$ of patients; in group 2 in $42.9 \%$ and $21.4 \%$, respectively; in group 3 in $31.3 \%$ and $29.7 \%$, respectively; and in group 4 in $47.2 \%$ and $67.7 \%$, respectively. After treatment, hemi-hypoesthesia and hemiparesis persisted in $27.1 \%$ and $50 \%$ of patients in group 1 ; in group 2 in $17.9 \%$ and $32.1 \%$; in group 3 in $31.3 \%$ and $12.5 \%$; and in group 4 in $18.1 \%$ and $41.7 \%$, accordingly. The regression of hemi-hypoesthesia was effective in $31.2 \%$ of patients in group 1; in group 2 in $11.6 \%$; in group 3 it was unchanged (31.3\%); in group 4 in $29.1 \%$. According to the effectiveness of treatment, EEG-BFB therapy, standard treatment, and Qigong therapy, respectively, yielded remarkable results. Acupuncture therapy yielded no results. The effectiveness of hemiparesis regression was $29.2 \%$ in group $1,8.9 \%$ in group $2,17.2 \%$ in group 3 , and $26 \%$ in group 4.

In group 1, numbness of extremities was observed in $56.3 \%$ of patients before and $12.5 \%$ after treatment; in group 2 in $42.9 \%$ before and $14.3 \%$ after treatment; in group 3 in $21.9 \%$ and $9.2 \%$, respectively; and in group 4 in $43.1 \%$ and $13.9 \%$, respectively. The regression efficiency was $43.7 \%$ in group $1 ; 28.6 \%$ in group $2,12.7 \%$ in group 3 , and $29.2 \%$ in group 4 . The most effective methods were EEG-BFB therapy, standard treatment, acupuncture, and Qigong therapy, respectively. Coordinator tests in the form of instability in the Romberg position, with impaired finger test, were detected in patients in group 1 in $29.2 \%$ and $39.6 \%$ before and $20.8 \%$ and $20.8 \%$ after treatment; in group 2 in $46.4 \%$ and $51.8 \%$ before and $16.1 \%$ and $14.3 \%$ after treatment; in group 3 in $53.1 \%$ and $45.3 \%$ before and $23.4 \%$ and $21.9 \%$ after treatment; and in group 4 in $29.2 \%$ and $31.9 \%$ before and in $25 \%$ and $13.9 \%$ after treatment. The efficiency of regression of instability in the Romberg position was $8.4 \%$ in group 1 ; $30.3 \%$ in group $2 ; 29.7 \%$ in group 3 ; and $4.2 \%$ in in group 4. The effectiveness of regression of the disturbance of the finger-bearing test was $18.8 \%$ in group $1 ; 37.5 \%$ in group $2 ; 33.4 \%$ in group 3 ; and $18 \%$ in group 4 . The treatment complexes with coordinative disorders in the form of instability in the Romberg position were effective in the use of Qigong therapy and acupuncture, while EEG-BFB therapy and standard treatment were less effective.

In group 1, unsteady gait was detected upon admission in $31.3 \%$ of patients and in $4.2 \%$ upon discharge; in group 2 - in $25 \%$ both upon admission and discharge; in group 3 in $42.2 \%$ and $9.2 \%$, respectively; and in group 4 in $23.6 \%$ and $12.5 \%$, respectively. The regression efficiency was $27.1 \%$ in group 1; unchanged in group $2(25 \%) ; 33 \%$ in group 3; and $11.1 \%$ in group 4 . Regression of violations of coordinating tests in the form of unsteady gait is noted when applying Qigong therapy, EEG-BFB therapy, or standard treatment. Acupuncture turned out to be com- 
pletely ineffective. Pathological symptoms of Babinsky and oral automatism were observed in $50 \%$ of patients in group 1 before treatment; $48.2 \%$ in group $2 ; 42 \%$ in group 3 ; and $63.9 \%$ in group 4 . After treatment, they were observed in $27.1 \%$ of patients in group 1 ; in $35.7 \%$ in group 2 ; in $20.4 \%$ in group 3 ; and in $54.1 \%$ in group 4 . The regression of pathological symptoms was effective in $22.9 \%$ of patients in group $1 ; 12.5 \%$ in group $2 ; 21.6 \%$ in group 3 ; and $9.8 \%$ in group 4. EEG-BFB therapy, Qigong therapy, acupuncture, and standard treatment proved to be the most effective for restoration of the pyramidal pathways, the pathological stop signs of which are signs of oral automatism.

The use of complex methods and a standard method of treatment contributes to a complete regression of general weakness. Recovery of cognitive impairment and their manifestations in the form of memory loss during treatment with Qigong therapy was $100 \%$, acupuncture $12.7 \%$, and EEG-BFB $10.4 \%$, and it was upon standard treatment $1.4 \%$. The effectiveness of regression of weakness in the limbs in patients receiving EEG-BFB therapy was $100 \%$, Qigong and the standard treatment were effective in $44.3 \%$ and $23.4 \%$ of patients, respectively. The effectiveness of treatment for a decrease in muscle tone caused by ischaemic stroke was observed in $17.8 \%$ of patients receiving the acupuncture treatment; in $13.9 \%$ receiving the standard method of treatment; in $12.5 \%$ receiving EEGBFB therapy; and in 12.3\% undergoing Qigong therapy. The effectiveness of treatment and regression of the Barre test was observed in $100 \%$ of patients treated with Qigong; $10.7 \%$ of those treated with acupuncture; no regression in those treated with EEG-BFB; and a 4.1\% increase after standard treatment. The effectiveness of the regression of pathological symptoms in the form of dysarthria was 56.2\% upon EEG-BFB treatment; it was $25 \%$ among patients who received acupuncture; $62.5 \%$ after Qigong therapy; and $100 \%$ regression was seen in the control group. A complete regression of clinical manifestations in the form of dysarthria was observed in the control group of patients who received standard treatment, then in the group of patients receiving Qigong therapy and EEG-BFB therapy. The Last place was taken by the group of patients who underwent acupuncture.

Articulation disorders upon EEG-BFB treatment amounted to $12.5 \%, 16 \%$ upon acupuncture, 9.3\% upon Qigong therapy, and remained unchanged in the control group (19.4\%). Regression of the articulation disorder was noted in the group of patients who received acupuncture, EEG-BFB, and Qigong therapy. The condition of patients in the control group remained unchanged. The visual impairment in the form of a weakness upon nystagmus, hemianopsia, anisocoria, and double vision was in the following order: group 2: $63.1 \%$; group 3: $29.1 \%$; group 1: $12.4 \%$; and group 4: 7\%. Notably, none of the treatment complexes contributed to the complete regression of these clinical manifestations. The comparison of effectiveness of medical complexes on the regression of clinical manifestations in the form of asymmetry of the nasolabial folds with a skewed face, paresis of the seventh and 12th pairs of craniocerebral, and bulbar disorders of the cranial nerves demonstrated a higher efficiency of EEG-BFB treatment (35.3\%), then acupuncture (21.7\%), Qigong therapy $(20.2 \%)$, and standard treatment, which proved to be least effective with an increase of $+4.1 \%$. The effectiveness of anisoreflexia regression upon EEGBFB therapy was 3\%, 16\% upon acupuncture, 5\% upon Qigong; and 17.9 upon standard treatment. The effectiveness of hemi-hypoesthesia regression was without changes in groups 1 to 3 , and treatment group 4 yielded no regression.

EEG-BFB therapy (31.2\%), standard treatment (29.1\%), Qigong therapy $(31.3 \%)$, and acupuncture treatment made no effect $(11.6 \%)$. The effectiveness of hemiparesis regression upon EEG-BFB training resulted in an increase in symptoms by $+29.2 \%$, upon acupuncture $8.9 \%$, upon Qigong therapy $17.2 \%$, and upon standard treatment $26 \%$. When treating hemiparesis, effective methods turned out to be as follows: standard treatment, Qigong, and acupuncture, with EEG-BFB therapy being the least effective method. The effectiveness of limb numbness regression was as follows: EEG-BFB therapy: 43.7\%, standard treatment: $29.2 \%$, acupuncture: $28.6 \%$, and Qigong therapy: $12.7 \%$. The efficiency of regression of instability in the Romberg's position was 30.3\% upon acupuncture, 29.7\% upon Qigong therapy, 8.4\% upon EEG-BFB treatment, and $4.2 \%$ upon standard treatment. The treatment complexes with coordinative disturbances in the form of instability in the Romberg's position were effective upon acupuncture in $37.5 \%$ of patients, upon Qigong therapy in $33.4 \%$, upon EEG-BFB therapy in $18.8 \%$, and least effective upon standard treatment in $18 \%$. Regression of coordination dysfunction in the form of instability when walking was observed upon Qigong therapy in 33\% of patients, upon EEG-BFB therapy in $27.1 \%$, and upon standard treatment in $11.1 \%$. The least efficiency was observed upon acupuncture, which yielded no changes (25\%); EEG-BFB therapy resulted in $22.9 \%$, Qigong therapy in $21.6 \%$, acupuncture in $12.5 \%$, and standard treatment in $9.8 \%$ proving to be the most effective method for restoring the function of the corticospinal tract, the pathological stop signs of which are indicative of oral automatism.

Notable regression of headaches was observed upon Qigong therapy, with $100 \%$ regression, upon acupuncture with $55.4 \%$, upon EEG-BFB therapy with $43.6 \%$, in the control group with $19.4 \%$. The effectiveness of nausea regression upon EEG-BFB treatment and Qigong therapy was $100 \%$, it was $46.2 \%$ upon acupuncture, and $19.3 \%$ upon standard treatment. The regression of nausea upon EEG-BFB treatment and Qigong was $100 \%$, and the use of acupuncture and standard treatment was effective in $30.2 \%$ and $8.4 \%$, respectively. A remarkable effect on nausea was noted upon EEG-BFB therapy and Qigong therapy. The use of acupuncture and standard treatment was less effective. Dizziness was registered in $64.6 \%$ of patients in group 1, in 59\% of patients in group 2, 65.6\% in group 3, and $34.8 \%$ in group 4 . The most effective treatments against dizziness were EEG-BFB, acupuncture, and Qigong therapy. The effectiveness of Qigong therapy was $100 \%$, acupuncture, $71.8 \%$; EEG-BFB, $52 \%$, and 
standard treatment in 7.8\%. Qigong gymnastics, acupuncture, and EEG-BFB methods were more effective upon removing patients from the state of inhibition during complex treatment compared with the control group of patients who remained in the state of inhibition in a significant amount. The effectiveness of the regression of general weakness was $100 \%$ in all 4 groups.

\section{Discussion}

The main aspect of poststroke neuroplasticity is cortical reorganisation. Neuronal plasticity and functional restoration lead to structural changes in the brain, called sprouting of dendrites and axons in the perifocal zone and distant parts of the brain, synaptogenesis (starting from the third day and reaches its highest point by the 7 th-14th day), angiogenesis develops a network of collateral vessels and promotes the migration of neuronal stem cells from the subventricular zone to the region of myocardial infarction. The leading role in neuroplasticity is played by cerebral neurotrophic factor, a representative of the family of nerve growth factor $(29,30)$.

According to the results of the Cochrane review, mirror therapy is an effective method for impaired hand function after ischaemic stroke (31). One-sided spatial neglect is a violation of perception, attention, and action in the space opposite to the affected hemisphere of the brain (usually in the left). Neglect occurs in most patients with stroke, causing damage to the temporoparietal sections (32). Prismatic adaptation is a method in which the plasticity of sensorimotor functions is stimulated by manipulation with sensorimotor relationships. The severity of spatial neglect decreases with prismatic therapy, which activates the associative zones of the cerebral cortex. In this method, the patient makes targeted movements with their hands and head to the designated visual stimulus, while the patient is in special glasses that shift the field of view of each eye to the right. When the patient takes out an object, a visual shift leads to mimic falling with a deviation to the right, the patient adapts over time. Positive results of prismatic adaptation are given in several meta-analyses $(33,34)$. During rehabilitation, to prevent the development of hemiplegic contracture in patients with ischaemic stroke, treatment by the position is used (35). Using the standard method of localising points provides efficiency compared with other known methods and increases the probability of detecting biologically active points that have a targeted selective therapeutic effect on a diseased organ and reduces redundancy in the choice of points (36-38).

According to the results of this study, a restoration of the bioelectric potentials of the brain in the main group of patients with $\alpha$ a and $\beta$ rhythms is observed mainly in the left hemisphere and a decrease in $\tau$ and $\delta$ potentials in both hemispheres. Changes in brain potentials with closed eyes after training are indicative of neuroplasticity processes due to inhibition of certain parts of the brain and the occurrence of levelling inhibition. In the control group, after standard treatment, insignificant changes in the EEG potentials were observed, which indicates the absence of training and the slowdown of the processes of repair of brain structures due to a decrease in the level of neuroplasticity.

Research (39) confirmed that Qigong exercises cause protective inhibition, which leads to a decrease in lactic acid in the arteries, a decrease in metabolic rate, and a decrease in plasma renin level. Many low-frequency alpha rhythms appear on the EEG with synchronisation 3 times higher than usual. After exercise, the prolactin density in plasma increases, dopamine activity decreases, and the excretion of the cortin hormone decreases by $50 \%$. This leads to an improvement in the immune system functioning. Exercise not only causes protective inhibition of the brain, but also regulates the function of the autonomic nervous system through the regulation of respiration. The pathological focus in the brain present in various diseases associated with a pathological reflex with a pathological arc being replaced by the Qigong arc. This leads to the normal conduct of impulses from the central nervous system to internal organs and systems, which then causes the extinction of the pathological focus in the brain.

\section{Conclusion}

This study investigated the effects of various treatment complexes on clinical manifestations in patients with acute ischaemic stroke. The most effective methods of stopping dizziness were EEG-BFB therapy, acupuncture, and Qigong therapy. Qigong, acupuncture, and EEG-BFB contributed to a more effective treatment for removing patients from the state of inhibition during complex treatment compared with the control group, most of whom remained in the state of inhibition. When treating hemiparesis, effective methods turned out to be as follows: standard treatment in the control group of patients, Qigong, and acupuncture. In this study, Qigong therapy contributed to the complete restoration of memory for patients, partially recovers memory with standard treatment and to a lesser extent in patients treated with EEGBFB therapy and acupuncture. The use of EEG-BFB treatment was most effective against weakness in the limbs.

The most effective method for treating the clinical and neurological manifestations of acute ischaemic stroke was EEG-BFB therapy. The acupuncture method ranked second in terms of effectiveness. The third place belonged to Qigong therapy. The fourth place in terms of effectiveness belonged to standard treatment.

\section{Acknowledgment}

None.

\section{Conflict of Interests}

The authors declare that they have no competing interests.

\section{References}

1.Vereshchagin NV, Morgunov VA, Gulevskaya TS. Pathology of the brain in atherosclerosis and arterial hypertension. Moscow: Nauka, 1997.

2.Vilensky BS. Stroke: prevention, diagnosis and treatment. St. Petersburg: Nauka, 1999.

3. Vibers DO, Feigin VL, Brown RD. Guide to cerebrovascular 
diseases. Moscow: Nauka, 1999.

4.Gorbacheva FE, Skoromets AA, Yakhno NN. Diseases of the nervous system. A Guide for Doctors 1995;1:152-256.

5. Warlow ChP. Stroke. A practical guide for the management of patients. St. Petersburg: Nauka, 1998.

6.Adams RM, Victor M, Ropper AH. Principles of neurology. New York: McGraw-Hill Education, 1997.

7.Yatsu FM, Grotta JC, Pettigrew LC. Stroke. St. Louis: 100 Maxims, 1995.

8. Gusev EI, Skvortsova VI, Komissarova IA, Dambinova SA, Gnedovskaya EV, Raevsky KS, et al. The neuroprotective effect of glycine in the acute period of ischaemic stroke. Korsakov J Neurol Psych. 1999;2:12-20.

9. World Stroke Campaign. 2018 [cited 2020 Sept 10]. Available from: http://www.worldstrokecampaign.org/images/global_ stroke_bill_of_rights/English_GlobalBO-Rights_web.pdf.

10. Kadykov AS, Shakhparonova NV. Early rehabilitation of stroke patients. The role of drug therapy. Nerv Dis. 2014;1:224.

11. Seliverstov YuA. Modern approaches to the effective rehabilitation of stroke patients. Atmosphere. Nerv Dis. 2014;3:37-42

12. Kulikov VP, Bespalov AG, Yakushev NN. Effectiveness of training with hypercapnic hypoxia in the rehabilitation of ischaemic brain damage in an experiment. Herald Regener Med. 2008;2:59-61.

13. Tregub $\mathrm{P}$, Kulikov V, Bespalov A. Tolerance to acute hypoxia maximally increases in case of joint effect of normobaric hypoxia and permissive hypercapnia in rats. Pathophysiology. 2013;20(3):165-70.

14. Tregub PP, Kulikov VP, Bespalov AG, Vvedensky AJ, Osipov IS. Neuroprotective effects of individual or combined exposure to hypoxia and hypercapnia in the experiment. Bull Exper Biol Med. 2013;155(3):327-9.

15. Alekseeva TM. Hypercapnic-hypoxic breathing exercises as a potential way of rehabilitation treatment for stroke patients. Arterial Hypertens. 2019;25(2):134-42.

16. Balci BD. Vestibular rehabilitation in acute central vestibulopathy: a randomised controlled trial. J Vestibular Res. 2013;23(4, 5):259-67.

17. Brown KE. Physical therapy for central vestibular dysfunction. Arch Physical Med Rehab. 2006;87(1):76-81.

18. Van Dijk H, Jannink MJA, Hermens HJ. Effect of augmented feedback on motor function of the affected upper extremity in rehabilitation patients: a systematic review of randomised controlled trials. J Rehab Med. 2005;37(4):20211 .

19. Reddy RP. Silent epidemic: The effects of neurofeedback on quality-of-life. Indian J Psycholog Med. 2014;36(1):40-52.

20. Moreira MC. Use of virtual reality in gait recovery among post stroke patients - a systematic literature review. Disability and Rehabilitation: Assistive Technology 2013;8(5):357-62.

21. Laver K. Cochrane review: virtual reality for stroke rehabilitation. Eur J Physical Rehab Med. 2012;48(3):523-30.

22. Ekusheva EV, Komazov AA. Use of the Anika rehabilitation glove by patients after a stroke: possibilities for increasing functional recovery. Farmateka. 2019;26(13):30-3.

23. Sabut SK. Functional electrical stimulation of dorsiflexor muscle: effects on dorsiflexor strength, plantarflexor spasticity, and motor recovery in stroke patients. NeuroRehab. 2011;29(4):393-400.

24. Barwood CHS, Murdoch BE. rTMS as a treatment for neurogenic communication and swallowing disorders. Acta Neurolog Scand. 2013;127(2):77-91.

25. Hsu WY. Effects of repetitive transcranial magnetic stimulation on motor functions in patients with stroke: a metaanalysis. Stroke. 2012;43(7):1849-57.
26. Cumming TB. Very early mobilisation after stroke fasttracks return to walking: further results from the phase II AVERT randomised controlled trial. Stroke. 2011;42(1):1538.

27. Neuhaus AA. Neuroprotection in stroke: the importance of collaboration and reproducibility. Brain. 2017;140(8):2079-92.

28. Professional medical information system. 2020 [cited 2020 Sept 10]. Available from: https://diseases.medelement.com/.

29. Bernhardt J. Early rehabilitation after stroke. Cur Opinion Neurol. 2017;30(1):48-54.

30. Coleman ER. Early rehabilitation after stroke: a narrative review. Cur Atherosclerosis Rep. 2017;19(12):59.

31. Thieme H. Mirror therapy for improving motor function after stroke: update of a Cochrane review. Stroke. 2019;50(2):e26-e27.

32. Tatuene JK. Incidence, risk factors and anatomy of peripersonal visuospatial neglect in acute stroke. Eur Neurol. 2016;75(3-4):157-63.

33. Barrett AM, Houston KE. Update on the clinical approach to spatial neglect. Cur Neurol Neuroscience Rep. 2019;19(5):147-54.

34. Champod AS. The effects of prism adaptation on daily life activities in patients with visuospatial neglect: a systematic review. Neuropsycholog Rehab. 2018;28(4):491-514.

35. Urban PP. Occurence and clinical predictors of spasticity after ischaemic stroke. Stroke. 2:010;41(9):2016-20.

36. Tabeeva DM. A practical guide to acupuncture. Moscow: MEDpress-Inform, 2014.

37. Bogdanov NN. Physiological characteristics of biologically active points. Physiology: Human. 1979;5(1):186-92.

38. Lien Zh. The guide to modern Zhen-Jiu therapy. Moscow: Nauka, 1959

39. Benson H. The relaxation response. New York: Harper Collins Publishers, 1975. 